\title{
Response of patients with hemodialysis- associated pruritus to new treatment algorithm with nalfurafine hydrochloride: a retrospective survey-based study
}

Naoko Takahashi ${ }^{1 *}$, Taku Yoshizawa ${ }^{1}$, Junko Kumagai ${ }^{1}$, Hideki Kawanishi ${ }^{2}$, Misaki Moriishi $^{3}$, Takao Masaki $^{4}$ and Shinichiro Tsuchiya ${ }^{2}$

\begin{abstract}
Background: Pruritus in hemodialysis patients is a serious complication that lowers quality of life and adversely affects survival. Hemodialysis-associated pruritus has multiple causes and treatments; however, no effective treatment has been established and many cases are treatment-resistant. Nalfurafine hydrochloride, introduced in 2009 as a treatment for hemodialysis-associated pruritus, has been shown to alleviate itching in hemodialysis patients with existing therapy-resistant pruritus. Based on those reports, we reviewed our strategy for pruritus management in hemodialysis patients in June 2009 and established a new treatment algorithm, which included nalfurafine hydrochloride, modified drug therapy, and patient guidance on skin care and daily life.
\end{abstract}

Methods: To evaluate the effectiveness of the new treatment algorithm, we conducted pre- and post-implementation surveys of 215 patients receiving maintenance hemodialysis. The first survey was performed in May 2009, before introduction of the algorithm, and the second in April 2013, 4 years after algorithm introduction. In both surveys, patients provided information about the presence of pruritus, its intensity, and their satisfaction with treatment.

Results: The percentage of patients with pruritus decreased significantly from 83.3 to $69.8 \%$ following algorithm introduction, accompanied by a significant reduction in the percentage of patients reporting severe itching (from 13.0 to $2.8 \%$ ). Furthermore, the percentage of patients with pruritus-related insomnia decreased from 12.6 to $4.7 \%$. Patient satisfaction with treatment improved significantly from 49.1 to $70.6 \%$. When analysis was confined to patients receiving nalfurafine hydrochloride as a component of pruritus management, the percentage of patients complaining of severe itching decreased from 46.7 to $0 \%$ after the start of treatment.

Conclusions: Our findings suggest that pruritus in hemodialysis patients can be improved with the introduction of a team-based treatment algorithm, consisting of drug therapy (including nalfurafine treatment), skin care, and daily-life guidance.

Keywords: End-stage renal disease, Hemodialysis, Kappa opioid receptor, Nalfurafine hydrochloride, Pruritus, Quality of life

\footnotetext{
* Correspondence: n-takahashi@tsuchiya-hp.jp

${ }^{1}$ Akane-Foundation Omachi Tsuchiya Clinic, 2-8-35 Omachi Higashi, Asa

Minami-Ku, Hiroshima 731-0124, Japan

Full list of author information is available at the end of the article
} 


\section{Background}

The number of chronic dialysis patients in Japan was approximately 314,000 at the end of 2013, and this number continues to grow annually [1]. Pruritus, which reduces quality of life (QOL), develops in 60-80 \% of hemodialysis patients [2-4]. Yamada et al. reported that 1289 (66.9\%) of 1927 hemodialysis patients surveyed in 2009 suffered from pruritus and that 461 (41.2\%) of 1120 hemodialysis patients had pruritus-related sleep disorders [2]. In a survey of 2550 hemodialysis patients, Omori et al. found that sleep disorders were more likely in patients with severe pruritus [3]. Not only is pruritus known to cause sleep disorders but can also contribute to the development of depression and has been associated with reduced survival rates. The reported prevalence of depression is 1.3-1.7 times higher among patients with moderate to severe pruritus-related disturbances in daily life, compared with patients not bothered by pruritus [4], and a negative correlation between pruritus intensity and survival has been demonstrated [5].

The etiology of pruritus in hemodialysis patients generally involves three types of abnormalities: (1) abnormalities originating from renal failure and hemodialysis; (2) skin abnormalities; and (3) abnormalities in the regulation of itch sensation by the central nervous system. The standard strategy for treating hemodialysis-associated pruritus involves combining therapies to address these three etiologic pathways.

Nalfurafine hydrochloride, a selective $\mathrm{k}$-opioid receptor agonist, was developed to treat intractable pruritus in hemodialysis patients. The efficacy and safety of nalfurafine were demonstrated in a prospective, randomized, double-blind comparative study and in a prospective, open-label, 52-week long-term dosing study in Japan [6, 7]. In March 2009, nalfurafine was first marketed in Japan to alleviate existing therapy-resistant pruritus in hemodialysis patients.

When nalfurafine became available for clinical use, our institution reviewed its strategy for managing pruritus in hemodialysis patients and introduced a new treatment algorithm. To verify the efficacy of the algorithm, we administered a questionnaire regarding pruritus twice to patients receiving maintenance hemodialysis, once before the introduction of the treatment algorithm, and again 4 years after algorithm introduction.

\section{Methods}

We performed a retrospective analysis of 215 patients (126 men and 89 women; mean age at the time of the second survey: $68.3 \pm 11.6$ years; mean duration of hemodialysis at the time of the second survey: $10.3 \pm 5.1$ years) who received ongoing maintenance hemodialysis at our facility between May 2009 and April 2013 and who completed both the pre-implementation and post-implementation questionnaires. Almost all patients received in-clinic dialysis, three times per week, $4 \mathrm{~h}$ per day, both before and after implementation of the new treatment algorithm. This study was approved by the ethical review board at Tsuchiya General Hospital (reference number: E151221-4). The patients included in this study provided oral informed consent in advance, which was recorded in the medical record.

A team of health-care practitioners began a review of our therapeutic strategy in June 2009. Prior to the review, our standard treatment protocol for hemodialysisassociated pruritus included antihistamines and antiallergy agents. Strategies to decrease pruritus arising from renal failure and hemodialysis itself include (1) the use of polymethylmethacrylate membranes with high biocompatibility and protein-adsorbing capability and (2) predilution online hemodiafiltration, which effectively removes protein-bound uremic toxins. These strategies were applied in individual cases based on clinical data, including the presence of pruritus, both before and after implementation of the new algorithm. Patients did not routinely receive guidance regarding skin care and daily life. Our standard treatment protocol for pruritus in hemodialysis patients did not include moisturizers, although we did prescribe moisturizers to patients with obviously dry skin.

Our new algorithm consisted of drug therapy (including nalfurafine), countermeasures against possible causes of pruritus in individual cases, skin care, and guidance for daily life (Fig. 1). Based on the new algorithm, patients were treated with nalfurafine if their pruritus was not responsive to moisturizers with or without topical steroids or if topical steroid use was undesirable.

A questionnaire was administered twice to evaluate pruritus in all hemodialysis patients, once in May 2009, before review of our therapeutic strategy, and again in April 2013, after implementation of the new algorithm. The survey included questions about the presence or absence of pruritus; the severity of pruritus (evaluated with the visual analog scale (VAS) [8] and the Shiratori severity score for pruritus, which is the foundation for the Kawashima classification [9]); the frequency of pruritusrelated insomnia (1 day, 2-3 days, 3-4 days, or 5 or more days per week); and the extent of patient satisfaction with treatment. Surveys were completed and returned by patients independently, except in the case of visual or other impairment, in which case a medical provider assisted with completion. The response rate for the initial survey was $95.9 \%$ (326/340 surveys completed); that of the second survey was $96.4 \%$ (396/411 surveys completed). In total, 215 patients completed both the initial and the second survey and were included in data analysis.

For the VAS, patients marked a labeled line of standardized length $(100 \mathrm{~mm})$ to indicate their level of pruritus. The 


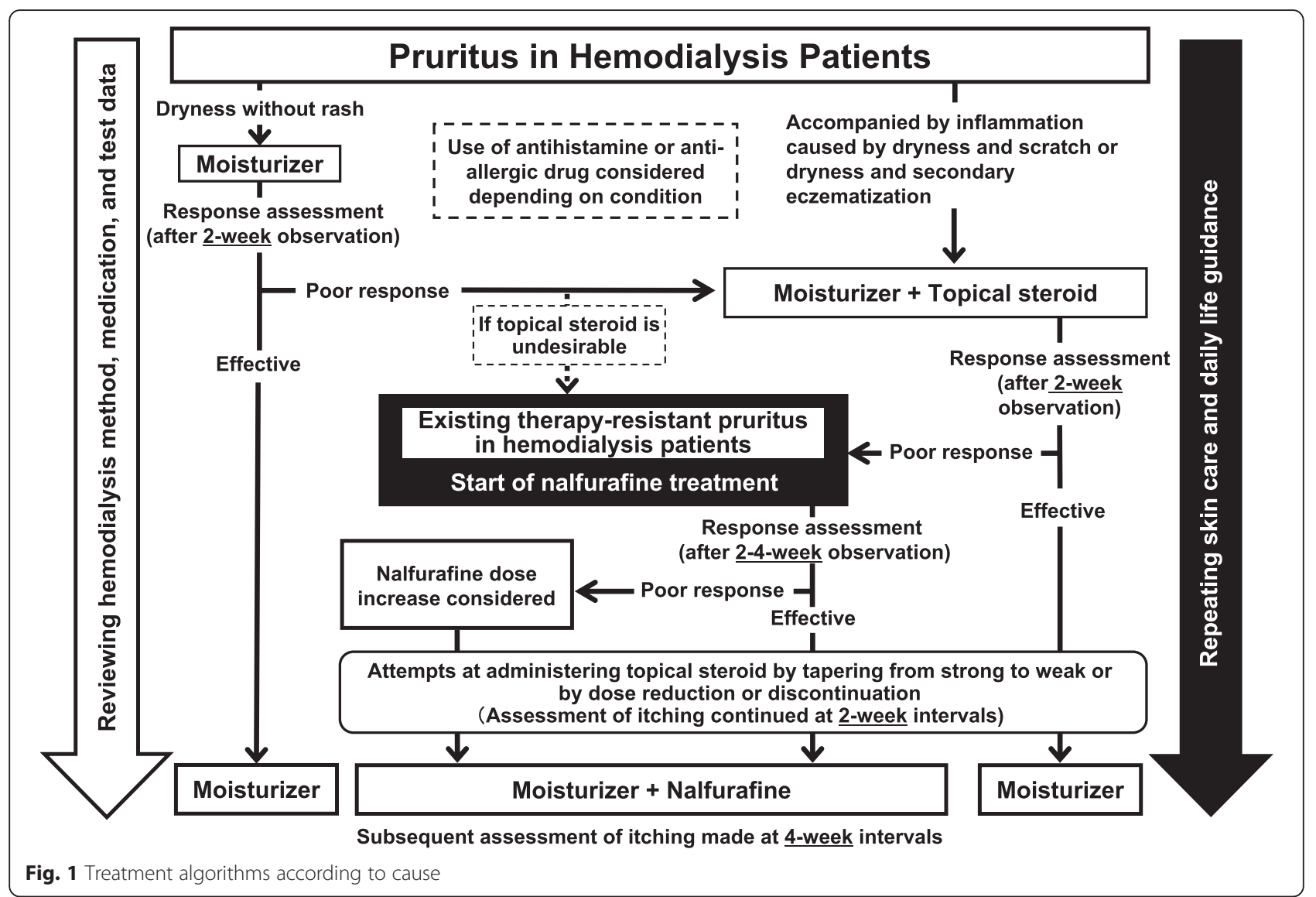

VAS score was determined based on the distance from the origin (no pruritus) to the patient's indicated mark. For the Shiratori severity score, daytime and nighttime pruritus were evaluated and scored separately. Daytime pruritus was rated on a five-grade scale: 0 (absent), 1 (endurable without scratching; minimal), 2 (subsides with slight scratching; mild), 3 (subsides with considerable scratching; moderate), or 4 (not subsiding with scratching, which prompts repeated scratching; severe). Nighttime pruritus was rated on a five-grade scale: 0 (absent), 1 (slight itching at bedtime but not causing intentional scratching; no difficulty sleeping because of pruritus), 2 (slight itching that subsides with scratching; no difficulty sleeping because of pruritus), 3 (difficulty sleeping because of pruritus that resolves with scratching; unconscious scratching occurs during sleep), or 4 (severe difficulty sleeping due to pruritus; frequent scratching that worsens pruritus). Grade 3 or higher on each scale was considered moderate to severe pruritus. The questionnaire asked patients who were prescribed medications for pruritus about their satisfaction with pruritus treatment and offered five possible responses: "very satisfied," "satisfied," "neutral," "dissatisfied," and "very dissatisfied."

In addition to the pruritus questionnaire, changes in the use of treatments such as moisturizers, oral antihistamines, topical antihistamines, topical steroids or other topical drugs, and anti-allergy injections were analyzed.

Shiratori severity scores and VAS values were expressed as the mean \pm standard deviation. The statistical significance of each parameter was evaluated with Wilcoxon signed-rank and paired $t$ tests. The presence or absence of pruritus, frequency of insomnia, and extent of treatment satisfaction were expressed as the number of patients that selected each category among all patients analyzed; the significance of differences in each parameter was analyzed with McNemar tests. In all analyses, $p$ values less than 0.05 were regarded as statistically significant. All statistical analyses were performed with JMP 10.0.2 statistical software (SAS Institute Inc., Cary, NC, USA).

\section{Results}

Presence and severity of pruritus

The number of patients who reported pruritus decreased significantly from 179 of 215 patients $(83.3 \%)$ before algorithm implementation to 150 of $215(69.8 \%)$ after implementation $(p<0.0001$, Fig. 2$)$. VAS values decreased significantly from $38.7 \pm 28.6 \mathrm{~mm}$ to $30.0 \pm 28.2 \mathrm{~mm} \mathrm{(} p=$ $0.0001)$. There was a significant decrease in the daytime Shiratori severity score from $2.05 \pm 1.20$ before algorithm introduction to $1.35 \pm 1.17$ after implementation $(p<$ 


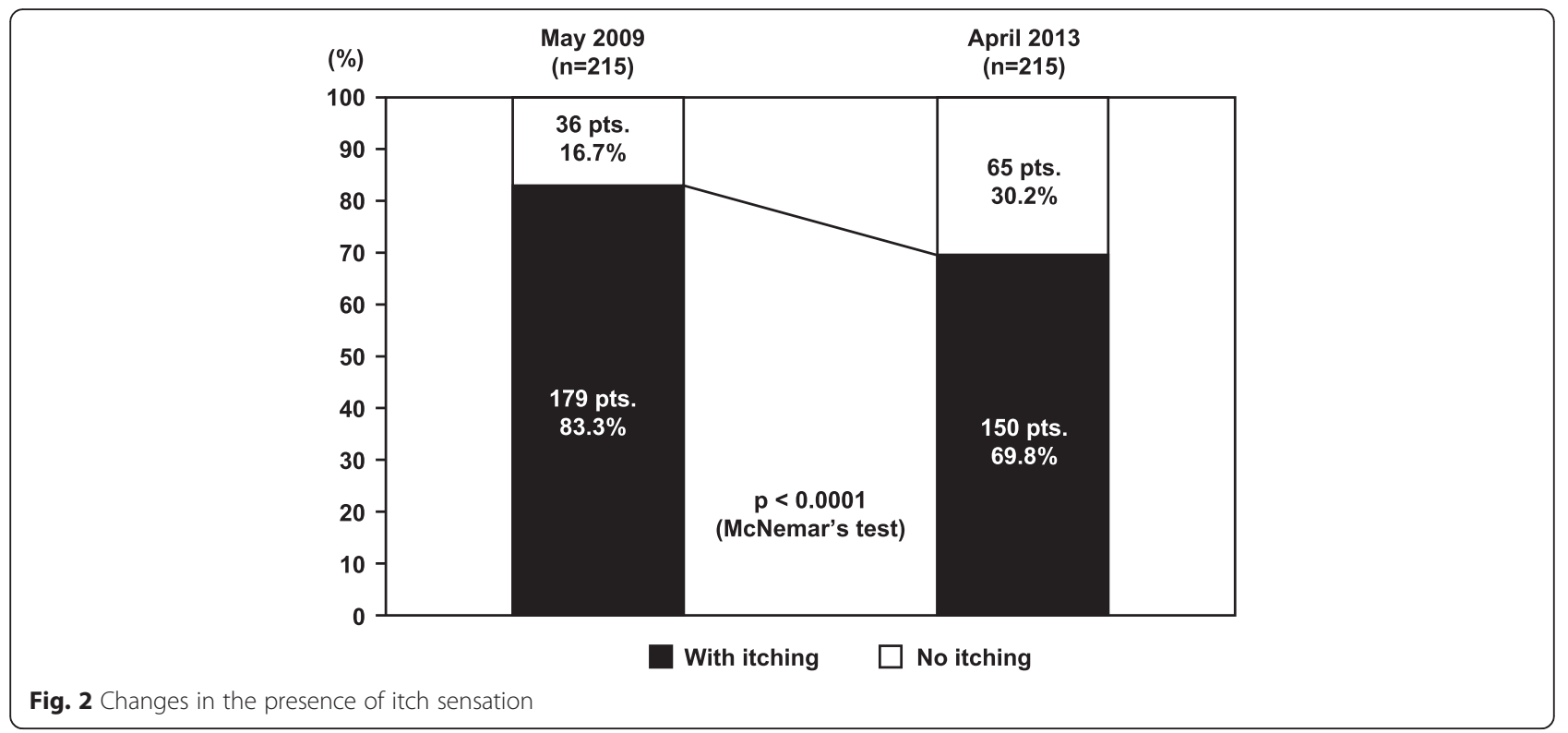

0.0001). The nighttime Shiratori score decreased from $1.26 \pm 1.10$ to $1.10 \pm 1.07$, although this difference was not statistically significant $(p=0.074)$.

The number of patients who reported moderate to severe pruritus (daytime Shiratori severity score of 3 or higher) decreased significantly from 67 of 215 patients (31.2\%) before algorithm introduction to 32 of 215 $(14.9 \%)$ after implementation $(p<0.0001)$. The number of patients who reported severe daytime pruritus (score 4) according to the Shiratori scoring system decreased significantly from 28 of $215(13.0 \%)$ to 6 of $215(2.8 \%)$ $(p<0.0001)$.
When the analysis was restricted to include only the 15 patients treated with nalfurafine, the VAS values decreased significantly from $69.1 \pm 19.5 \mathrm{~mm}$ before algorithm introduction to $34.1 \pm 22.8 \mathrm{~mm}$ after implementation $(p<0.005)$. This decrease was accompanied by a significant reduction in daytime Shiratori severity scores (from $3.40 \pm 0.63$ to $1.80 \pm 1.01 ; p=0.001$ ) and a significant reduction in nighttime Shiratori severity scores (from $2.33 \pm 1.11$ to $1.40 \pm 1.12 ; p<0.05$ ). Among the 15 patients treated with nalfurafine, the number who reported moderate to severe pruritus decreased significantly from $14(93.3 \%)$ to $4(26.7 \% ; p<0.005)$. The

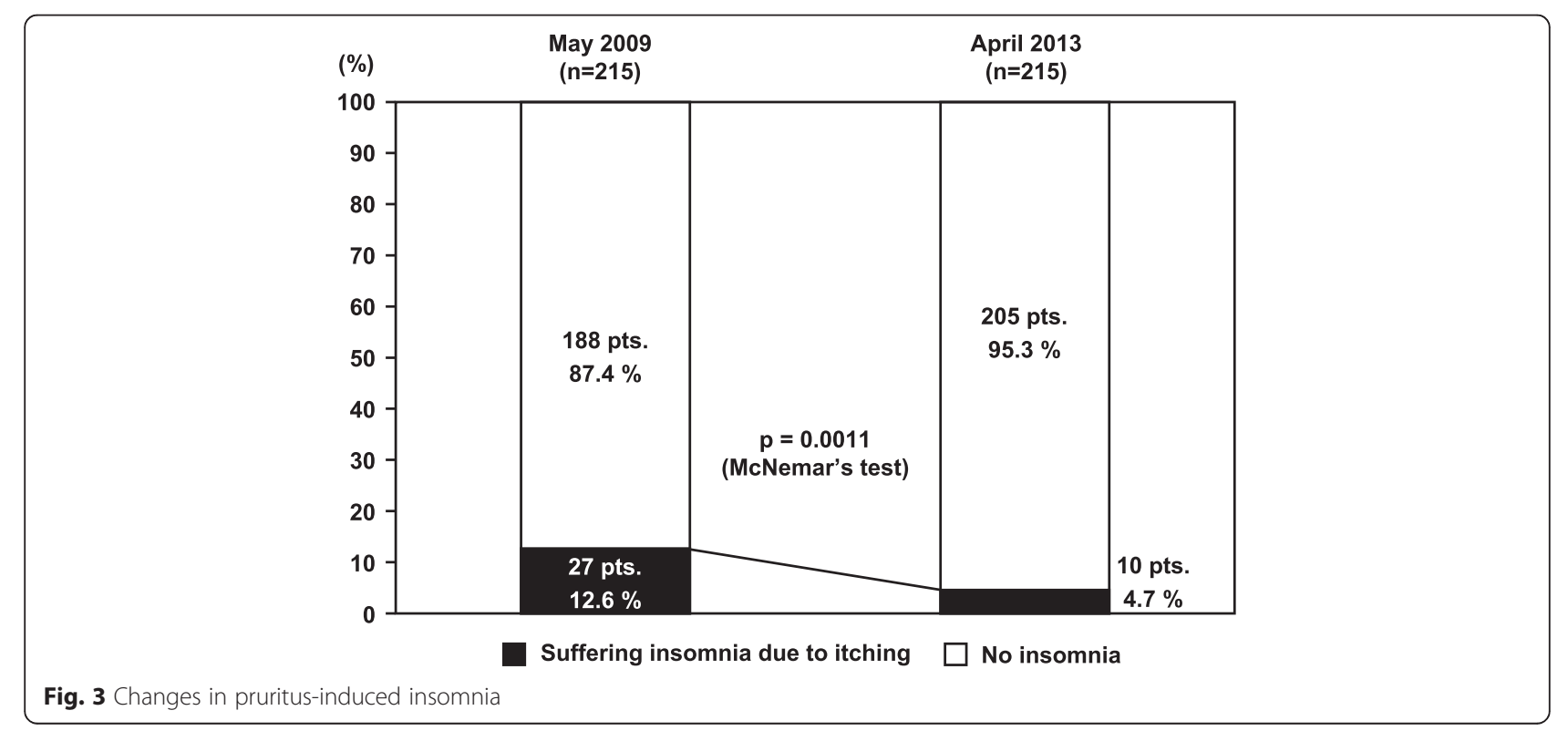


number who reported severe pruritus decreased from 7 $(46.7 \%)$ to none $(0 \%)$.

\section{Insomnia resulting from pruritus}

The number of patients with insomnia resulting from pruritus decreased significantly from 27 of 215 (12.6\%) before implementation of the treatment algorithm to 10 of $215(4.7 \%)$ after implementation ( $p=0.0011$; Fig. 3$)$.

\section{Changes in medications used for pruritus treatment}

An analysis of changes in the drugs used for pruritus treatment among patients who received medication at our facility revealed a marked increase in the percentage of patients who used moisturizers (from $31.9 \%$ before implementation to $60.8 \%$ after implementation). In contrast, there was a decrease in the percentage of patients who used oral antihistamines (from 46.9 to $27.7 \%$ ), topical antihistamines (from 6.4 to $4.8 \%$ ), topical steroids (from 46.9 to $43.2 \%$ ), other topical drugs (from 19.1 to $8.2 \%$ ), and in the number of patients who received antiallergy injections (from 23.5 to $4.8 \%$ ). Among patients treated with medications at our facility, $25.7 \%$ were receiving nalfurafine as of April 2013 (Fig. 4).

\section{Changes in use of hemodialysis-specific strategies to reduce pruritus}

There were no significant differences in the percentage of patients using PMMA membrane in 2009 (2.8 \%) versus 2013 (4.2\%; $p=0.3657$, McNemar test). Likewise, the percentage receiving online HDF did not change significantly between 2009 (7.4 \%) and 2013 (7.9\%; $p=0.6547$, McNemar test).

\section{Changes in $\mathrm{Ca} / \mathrm{IP}$ status}

Data on calcium and phosphorus levels were available for 214 of the 215 participants. The percentage of patients whose corrected Ca level was within the target control range was significantly higher in 2009 (89.7 \%) than in $2013(80.8 \% ; p=0.0046)$, while the percentage of patients with IP levels within the target control range did not differ significantly between the 2 years $(75.7 \%$ in 2009 versus $73.8 \%$ in 2013; $p=0.6326$ ). The percentage of patients whose corrected $\mathrm{Ca} \times \mathrm{IP}$ product was within the target control range was significantly higher in 2013 than in 2009 (85.1\% in 2013 versus $77.6 \%$ in 2009; $p=$ 0.0325). However, when we used a chi-square test to analyze the correlations (1) between corrected $\mathrm{Ca} \times \mathrm{IP}$ product and moderate or severe pruritus and (2) between corrected $\mathrm{Ca} \times \mathrm{IP}$ product and presence/absence of pruritus, no significant correlations were found, suggesting that $\mathrm{Ca} \times \mathrm{IP}$ product did not affect the presence/ absence or severity of pruritus.

\section{Treatment satisfaction}

In May 2009, less than half of patients reported that they were "very satisfied" or "satisfied" with treatment (25 of $51,49.1 \%)$; this percentage increased significantly to 36 of 51 (70.6 \%) in April 2013 ( $p<0.05$, Fig. 5).

\section{Discussion}

Pruritus develops in 60-80\% of hemodialysis patients [2-4] as a result of abnormalities originating from renal failure and hemodialysis itself, skin abnormalities, and abnormalities in the regulation of itch sensation by the central nervous system (Fig. 6). The complex interplay

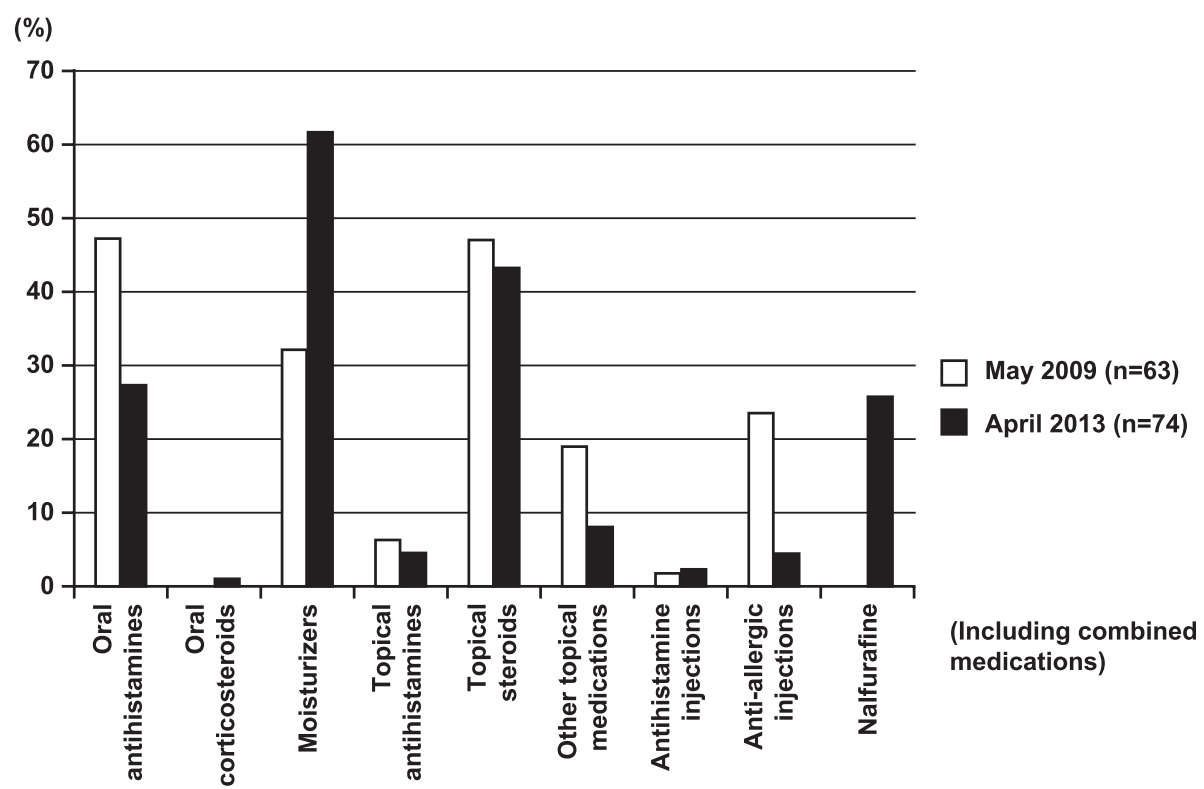

Fig. 4 Changes in drugs used for pruritus treatment in patients managed at our facility 


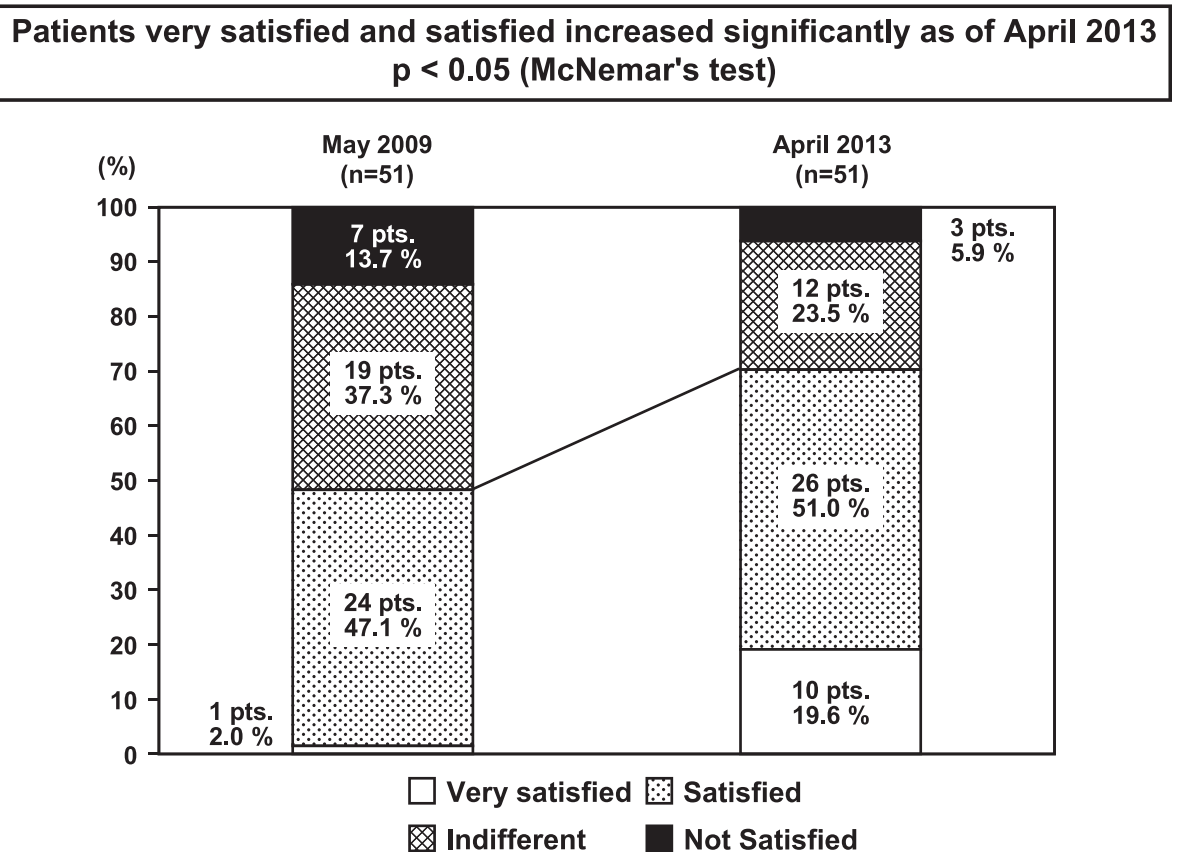

Fig. 5 Changes in patient satisfaction with treatment

of these multiple factors in hemodialysis patients can result in pruritus that often fails to respond sufficiently to existing treatments, including antihistamines, antiallergy drugs, and steroids [10-13].

The first type of abnormality, those resulting from renal failure or hemodialysis itself, is thought to involve the accumulation of uremic substances, elevation of serum calcium and phosphorus levels, secondary hyperparathyroidism, influences from substances that initiate pruritus (e.g., histamine and substance P), and the activation of complement and interleukins by hemodialysis membranes. The second type, skin abnormalities, includes dry skin and growth of nerve fibers (C fibers) into the epidermis as a result of dryness. Dryness itself may be caused by multiple factors, including lower stratum corneum moisture, decreased sweating, and reduced sebaceous gland secretion.

In the third type of abnormality, abnormal itch sensation regulation by the central nervous system, the possible role of endogenous opioids has recently received increased attention. A previous report indicated that hemodialysis patients with intense pruritus had a higher

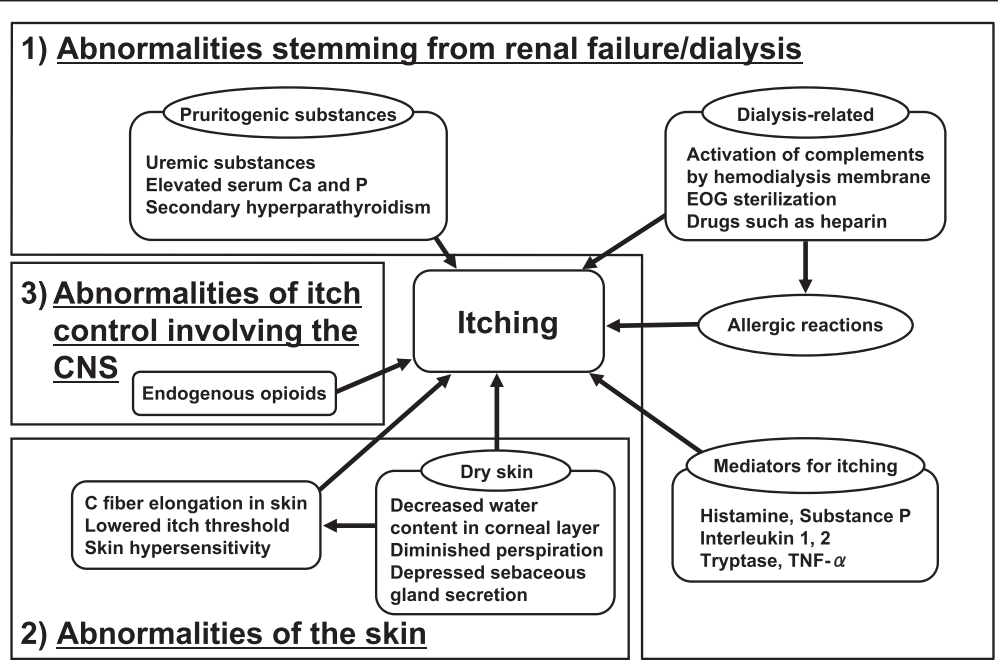

Fig. 6 Causes of pruritus in hemodialysis patients 
ratio of endogenous $\mu$-opioid peptide to endogenous $\kappa$-opioid peptide in the serum, suggesting that $\mu$-opioids induced pruritus while $\kappa$-opioids suppressed the condition [14]. Nalfurafine, a selective $\mathrm{k}$-opioid receptor agonist, was introduced in 2009 to address central nervous systemmediated therapy-resistant pruritus. Our institution introduced the use of nalfurafine as part of a new treatment algorithm for pruritus in hemodialysis patients after conducting a review of our existing treatment protocol.

We surveyed hemodialysis patients twice, once before the review of our pruritus treatment strategy, and again approximately 4 years after implementation of the new algorithm. Because of the possible psychological impact of switching from existing therapies for pruritus to new treatment methods and the reliance on patient selfassessment regarding pruritus severity, the second survey was administered approximately 4 years following review of our previous treatment strategy. The validity of selecting this time period has not been verified.

Our pre-algorithm survey results, which revealed a high prevalence of pruritus and low satisfaction with treatment among hemodialysis patients, are in agreement with those of a large-scale survey conducted by Yamada et al. that included 858 patients [2]. That study reported that $54.8 \%$ of patients were satisfied or very satisfied with their pruritus treatment, while $45.2 \%$ were neutral, dissatisfied, or very dissatisfied with treatment. In the present study, the prevalence of pruritus decreased and patient satisfaction with treatment increased after implementation of the new treatment protocol. Furthermore, the daytime Shiratori severity scores decreased significantly. The nighttime Shiratori severity scores also decreased, although the change was not statistically significant. Prior to changes in our pruritus treatment strategy, $31.2 \%$ of patients reported moderate to severe daytime pruritus according to the Shiratori criteria and $12.1 \%$ reported severe nighttime pruritus; mean Shiratori severity scores were $2.05 \pm 1.20$ during the day and $1.26 \pm$ 1.10 at night. Thus, before changes in our treatment strategy, pruritus tended to be less severe at night than during the day. This difference helps to explain why changes in our treatment strategy did not result in significant differences in nighttime pruritus. However, the percentage of patients who reported insomnia resulting from pruritus did decrease significantly.

Implementation of the new treatment algorithm resulted in decreased use of oral antihistamines, topical antihistamines, topical steroids, other topical drugs, and anti-allergy injections. In the past, antihistamines and anti-allergy drugs were often prescribed even for patients without dermatological conditions, in the absence of valid pruritus treatments. After reviewing our pruritus treatment strategy and the efficacy of these drugs, the use of antihistamines and anti-allergy drugs was restricted to patients who were diagnosed with dermatological diseases requiring these therapies. This change probably explains the decrease in the use of existing drugs (Fig. 1).

Between the first survey in 2009 and the second in 2013, 58 of the surveyed hemodialysis patients died. The data from these patients was not included in our analyses, which raises the concern that our data are valid only for hemodialysis patients with a relatively good prognosis. However, analysis of our initial survey data revealed that the prevalence of pruritus and the mean daytime Shiratori score were significantly lower $(p=0.003$ and $p=0.027$, respectively) and the prevalence of moderate or severe pruritus, mean VAS scores, and mean nocturnal Shiratori scores were lower (although not significantly so) among these excluded patients. Therefore, it is unlikely that the severity of pruritus at the time of the 2009 questionnaire was higher among the excluded patients than among those included. We therefore consider it unlikely that exclusion of the 58 deceased patients from analysis resulted in overestimation of the efficacy of this new treatment algorithm.

The efficacy of 52 weeks of treatment with nalfurafine was demonstrated in a pre-marketing long-term dosing study [7]. At our facility, nalfurafine treatment for 104 weeks in 13 patients with therapy-resistant pruritus resulted in a significant reduction in VAS values during the treatment period [15]. These results indicate that continuous treatment with nalfurafine alleviates pruritus over a long period in hemodialysis patients. In a previous study of patients treated with nalfurafine, the authors found that 6 out of 7 patients were able to discontinue existing oral drug therapy and to reduce the dose of topical steroids, switch to a less potent topical steroid, or discontinue topical steroids altogether [16]. Yamada et al. reported that $42.3 \%$ of patients treated with nalfurafine experienced alleviation of nighttime pruritus and sleep disorders [2]. Inui et al. reported that treatment with nalfurafine for 4 weeks resulted in a significant reduction in the mean VAS values and in State-Trait Anxiety Inventory scores for state anxiety [17]. Thus, nalfurafine has been shown to be useful for improving state anxiety in hemodialysis patients [18].

One possible explanation for the low satisfaction of hemodialysis patients with pruritus treatment is that in many patients, pruritus involves a complex interplay among multiple factors, which can cause therapeutic resistance. In the present study, nalfurafine suppressed existing therapy-resistant pruritus and enabled dose reduction or discontinuation of ineffective therapies, suggesting that nalfurafine can significantly contribute to improved QOL by alleviating sleep disorders and anxiety. We therefore conclude that including oral nalfurafine in the treatment of therapy-resistant pruritus at our facility has been very important clinically. 
To implement this treatment algorithm, a team-based approach to patient care is essential. At our facility, team-based treatment has been performed in the following way. Nurses provide guidance to patients regarding skin care and daily life, pharmacists supply information regarding the correct use of medications, clinical engineers carry out meticulous management of various data obtained from usual medical care, and physicians make final assessments regarding treatment response. It is not possible to treat pruritus with medications alone. The team-based approach, which involves health-care professionals, the patient, and the patient's family, is important so that patients with pruritus can be sufficiently monitored and encouraged to continue the use of supplemental agents such as daily moisturizer. This type of team-based approach is expected to improve pruritus awareness among health-care professionals and patients and to improve patient outcomes.

This study had several limitations. First, there was no control group. Therefore, it is difficult to certify that the new treatment algorithm was the most important factor in alleviating pruritus. Second, we did not investigate the change in patient QOL, although we did investigate changes in pruritus-induced insomnia and patient satisfaction with treatment. It remains uncertain whether the new treatment algorithm improves patient QOL. Third, the second survey was administered approximately 4 years after the review of our previous treatment strategy, to avoid effects from the psychological impact of switching treatment methods. The validity of selecting this time period has not been verified. Finally, it was not possible to identify the cause of pruritus in individual patients because hemodialysis-associated pruritus involves the complex interplay among multiple factors.

\section{Conclusions}

The recently introduced pruritus treatment algorithm for hemodialysis patients at our facility, consisting of drug therapy (including nalfurafine), skin care, and guidance for daily living, effectively alleviated therapy-resistant pruritus, decreased pruritus-associated insomnia, reduced the use of antipruritic medications, and increased patient satisfaction with treatment. Further prospective studies are needed to confirm the roles of each treatment intervention in a comprehensive approach to hemodialysisassociated pruritus.

\section{Competing interests}

The authors declare that they have no competing interests.

\section{Authors' contributions}

NT designed the new treatment algorithm and drafted the manuscript. TY participated in the design of the study and performed the statistical analysis. JK, HK, MM, TM, and ST conceived the study, participated in its design and coordination, and helped to draft the manuscript. All authors read and approved the final manuscript.

\section{Author details}

Akane-Foundation Omachi Tsuchiya Clinic, 2-8-35 Omachi Higashi, Asa Minami-Ku, Hiroshima 731-0124, Japan. ${ }^{2}$ Department of Artificial Organs, Akane-Foundation, Tsuchiya General Hospital, Hiroshima, Japan.

${ }^{3}$ Akane-Foundation Nakajima Tsuchiya Clinic, Hiroshima, Japan. ${ }^{4}$ Department of Nephrology, Hiroshima University Hospital, Hiroshima, Japan.

Received: 12 November 2015 Accepted: 31 March 2016

Published online: 30 May 2016

References

1. Masakane I, Nakai S, Ogata S, Kimata N, Hanafusa N, Hamano T, et al. An overview of regular dialysis treatment in Japan (As of December 31, 2013). J Jpn Soc Dial Ther. 2015;48:1-32 (In Japanese).

2. Yamada S, Sakurai $H$, Kasuga $H$, Kawahara $H$. Investigation of the status of uremic pruritus in hemodialysis patients and the efficacy of nalfurafine hydrochloride-questionnaire administered to 1936 patients from 17 clinics in Tokai area of Japan. J Jpn Soc Dial Ther. 2012;45:1133-40 (In Japanese).

3. Omori $K$, Aoike I, Aoyagi $H$, Aoyagi R, Imai K, Iwafuchi $Y$, et al. Risk factors for uremic pruritus in long-term hemodialysis patients. J Jpn Soc Dial Ther. 2001;34:1469-77 (In Japanese).

4. Pisoni RL, Wikström B, Elder SJ, Akizawa T, Asano Y, Keen ML, et al. Pruritus in haemodialysis patients: international results from the Dialysis Outcomes and Practice Patterns Study (DOPPS). Nephrol Dial Transplant 2006;21:3495-505

5. Narita I, Alchi B, Omori K, Sato F, Ajiro J, Saga D, et al. Etiology and prognostic significance of severe uremic pruritus in chronic hemodialysis patients. Kidney Int. 2006;69:1626-32.

6. Kumagai H, Ebata T, Takamori K, Muramatsu T, Nakamoto H, Suzuki H. Effect of a novel kappa-receptor agonist, nalfurafine hydrochloride, on severe itch in 337 haemodialysis patients: a phase III, randomized, double-blind, placebo-controlled study. Nephrol Dial Transplant. 2010;25:1251-7.

7. Kumagai H, Ebata T, Takamori K, Miyasato K, Muramatsu T, Nakamoto H, et al. Efficacy and safety of a novel $\mathrm{k}$-agonist for managing intractable pruritus in dialysis patients. Am J Nephrol. 2012;36:175-83.

8. Wahlgren CF, Ekblom A, Hägermark O. Some aspects of the experimental induction and measurement of itch. Acta Derm Venereol. 1989;69:185-9.

9. Kawashima M, Tango $T$, Noguchi $T$, Inagi M, Nakagawa $H$, Harada S. Addition of fexofenadine to a topical corticosteroid reduces the pruritus associated with atopic dermatitis in a 1-week randomized, multicentre, double-blind, placebo-controlled, parallel-group study. Br J Dermatol. 2003;148:1212-21.

10. Massry SG, Popovtzer MM, Coburn JW, Makoff DL, Maxwell MH, Kleeman CR. Intractable pruritus as a manifestation of secondary hyperparathyroidism in uremia. Disappearance of pruritus after subtotal parathyroidectomy. N Engl Med. 1968;279:697-700.

11. Patel TS, Freedman BI, Yosipovitch G. An update on pruritus associated with CKD. Am J Kidney Dis. 2007:50:11-20.

12. Stockenhuber F, Kurz RW, Sertl K, Grimm G, Balcke P. Increased plasma histamine levels in uraemic pruritus. Clin Sci (Lond). 1990;79:477-82.

13. Kaku H, Fujita Y, Yago H, Naka F, Kawakubo H, Nishikawa K, et al. Study on pruritus in hemodialysis patients and the antipruritic effect of neurotropin: plasma levels of substance $\mathrm{P}$, somatostatin, IgE, PTH and histamine. Nihon Jinzo Gakkai Shi. 1990;32:319-26 (In Japanese).

14. Kumagai H, Saruta T, Matsukawa S, Utsumi J. Prospects for a novel k-opioid receptor agonist, TRK-820, in uremic pruritus. In: Yosipovitch G, Greaves MW, Fleischer JA, McGlone F, editors. Itch: basic mechanisms and therapy. New York: Marcel Dekker; 2004. p. 279-86.

15. Takahashi N, Yoshizawa T, Kumagai J. Efficacy and safety of long-term (104-week) administration of nalfurafine hydrochloride. J Jpn Soc Dial Ther. 2013:46(3):371-8 (In Japanese).

16. Yoshizawa T, Kumagai J, Takahashi N, Tsuchiya S. Nalfurafine hydrochloride enabled to reduce dosage or discontinue conventional treatment for uremic pruritus. Kidney Dial. 2013;75:457-64 (In Japanese).

17. Suzuki T, Tsukamoto K, Abe K. Characteristics factor structures of the Japanese version of the State-Trait Anxiety Inventory: coexistence of positive-negative and state-trait factor structures. J Pers Assess. 2000;74:447-58

18. Inui S, Shirakawa Y, Itami S. Effect of nalfurafine hydrochloride on pruritus and anxiety level in hemodialysis patients. J Dermatol. 2011;38:1-2. 